\title{
Density Fluctuations in the Quark-Gluon Plasma
}

\author{
Stanisław Mrówczyńskif \\ Sottan Institute for Nuclear Studies, \\ ul. Hoża 69, PL - 00-681 Warsaw, Poland \\ and Institute of Physics, Pedagogical University, \\ ul. Konopnickiej 15, PL - 25-406 Kielce, Poland
}

Using the kinetic theory we discuss how the particle and energy densities of the quark-gluon plasma fluctuate in a space-time cell. The fluctuations in the equilibrium plasma and in that one from the early stage of ultrarelativistic heavy-ion collisions are estimated. Within the physically interesting values of the parameters involved the fluctuations appear sizeable in both cases.

PACS: 12.38.M, 52.25.Gj, 25.75.+r

Keywords: Quark-gluon plasma; Fluctuations; Relativistic heavy-ion collisions

23-th May 1997

Revised 26-th August 1997

2-nd revision 24-th November 1997

\footnotetext{
${ }^{1}$ E-mail: MROW@FUW.EDU.PL
} 
The quark-gluon plasma, which is expected to be produced in energetic heavy-ion collisions, is usually described in terms of the averaged quantities like baryon or energy density, temperature, etc. However, it is often important to know how these quantities treated as local ones fluctuate around their average values. For example, it has been argued in our papers [1] that the color current fluctuating around zero can initiate the plasma instabilities. Gyulassy, Rischke and Zhang [2] have recently shown that the hydrodynamical evolution of the quark-gluon plasma can be significantly influenced by the energy density fluctuations at the initial state.

In this note we derive, using the kinetic theory methods, simple analytic expressions describing how the particle and energy densities fluctuate in a space-time cell of the volume $\Delta x^{3} \Delta t$. Then, we use the derived formulas to estimate the particle and energy density fluctuations in the equilibrium plasma and in the parton system from the early stage of ultrarelativistic heavy-ion collisions at RHIC or LHC. Since the approach, which is employed, is rather simplistic our results should be mainly treated as a guiding for a more quantitative study in the future.

Let us start with the fluctuations of particle density $\rho(x)$, where $x \equiv(t, \mathbf{x})$ is a four-position. The average density is expressed through the distribution function $f$ as

$$
\langle\rho(x)\rangle=\langle\rho\rangle=\int \frac{d^{3} p}{(2 \pi)^{3}} f(\mathbf{p}),
$$

where $\mathbf{p}$ is the particle momentum. The system is assumed be on average homogeneous and stationary and consequently, the distribution function is independent of $x$. The momentum distribution is arbitrary.

The density correlation function for the classical system of noninteracting particles is given by a well known formula, see e.g. [3], as

$$
A(x) \stackrel{\text { def }}{=}\left\langle\rho\left(x_{1}\right) \rho\left(x_{2}\right)\right\rangle-\langle\rho\rangle^{2}=\int \frac{d^{3} p}{(2 \pi)^{3}} f(\mathbf{p}) \delta^{(3)}\left(\mathbf{x}-\mathbf{v}_{p} t\right)
$$

where $x \equiv x_{1}-x_{2}=\left(t_{1}-t_{2}, \mathbf{x}_{1}-\mathbf{x}_{2}\right)$ and $\mathbf{v}_{p}$ is the particle velocity equal $\mathbf{p} / E_{p}$ with $E_{p} \equiv \sqrt{\mathbf{p}^{2}+m^{2}}$. Due to the average space-time homogeneity the correlation function $A(x)$ depends on the difference of $x_{1}$ and $x_{2}$ only. The space-time points $\left(t_{1}, \mathbf{x}_{1}\right)$ and $\left(t_{2}, \mathbf{x}_{2}\right)$ are correlated in the system of noninteracting particles if the particles fly from $\left(t_{1}, \mathbf{x}_{1}\right)$ to $\left(t_{2}, \mathbf{x}_{2}\right)$. For this reason the delta $\delta^{(3)}(\mathbf{x}-\mathbf{v} t)$ is present in the formula (2). The momentum integral of the distribution function simply represents the summation over particles. Applicability of our classical approach to a quantum system such as a quark-gluon plasma is considered below. We also discuss there when the partons can be taken as noninteracting as in eq. (2).

We get the particle density fluctuations in a given space-time cell averaging the correlation function $A(x)$ over the cell volume. Specifically,

$$
\left\langle\rho^{2}\right\rangle-\langle\rho\rangle^{2} \equiv \int d^{4} x \Delta(x) A(x),
$$


where the coarse-graining function $\Delta(x)$ is chosen to be of the gaussian shape i.e.

$$
\Delta(x)=\frac{1}{4 \pi^{2} \Delta t \Delta x^{3}} \exp \left(-\frac{t^{2}}{2 \Delta t^{2}}-\frac{\mathbf{x}^{2}}{2 \Delta x^{2}}\right)
$$

with $\Delta t$ and $\Delta x$ denoting the root mean square of the cell size in time and space direction, respectively.

Substituting the correlation function (2) into eq. (3) we get

$$
\left\langle\rho^{2}\right\rangle-\langle\rho\rangle^{2}=\frac{1}{(2 \pi)^{3 / 2} \Delta x^{2}} \int \frac{d^{3} p}{(2 \pi)^{3}} \frac{f(\mathbf{p})}{\sqrt{\Delta x^{2}+\mathbf{v}_{p}^{2} \Delta t^{2}}} .
$$

As seen, the fluctuations remain finite for $\Delta t=0$ but grow to infinity when $\Delta x \rightarrow 0$.

For massless particles $\mathbf{v}_{p}^{2}=1$ and the formula (四) essentially simplifies. The relative fluctuations then are

$$
\frac{\left\langle\rho^{2}\right\rangle-\langle\rho\rangle^{2}}{\langle\rho\rangle^{2}}=\frac{1}{(2 \pi)^{3 / 2} \Delta x^{2} \sqrt{\Delta x^{2}+\Delta t^{2}}} \frac{1}{\langle\rho\rangle},
$$

and depend only on the average density and the size of the space-time cell. In the case of massive particles, a similar simplification occurs for $\Delta t=0$.

One easily generalizes the above considerations to the energy density fluctuations. The average energy density and the respective correlation function read

$$
\begin{gathered}
\langle\varepsilon\rangle=\int \frac{d^{3} p}{(2 \pi)^{3}} E_{p} f(\mathbf{p}), \\
W(x) \stackrel{\text { def }}{=}\left\langle\varepsilon\left(x_{1}\right) \varepsilon\left(x_{2}\right)\right\rangle-\langle\varepsilon\rangle^{2}=\int \frac{d^{3} p}{(2 \pi)^{3}} E_{p}^{2} f(\mathbf{p}) \delta^{(3)}\left(\mathbf{x}-\mathbf{v}_{p} t\right) .
\end{gathered}
$$

One finds the energy density fluctuations in the space-time cell as

$$
\left\langle\varepsilon^{2}\right\rangle-\langle\varepsilon\rangle^{2} \equiv \int d^{4} x \Delta(x) W(x)=\frac{1}{(2 \pi)^{3 / 2} \Delta x^{2}} \int \frac{d^{3} p}{(2 \pi)^{3}} \frac{E_{p}^{2} f(\mathbf{p})}{\sqrt{\Delta x^{2}+\mathbf{v}_{p}^{2} \Delta t^{2}}}
$$

For massless particles the relative energy density fluctuations are

$$
\frac{\left\langle\varepsilon^{2}\right\rangle-\langle\varepsilon\rangle^{2}}{\langle\varepsilon\rangle^{2}}=\frac{1}{(2 \pi)^{3 / 2} \Delta x^{2} \sqrt{\Delta x^{2}+\Delta t^{2}}} \frac{\left\langle e^{2}\right\rangle}{\langle\varepsilon\rangle^{2}},
$$

with

$$
\left\langle e^{2}\right\rangle \equiv \int \frac{d^{3} p}{(2 \pi)^{3}} E_{p}^{2} f(\mathbf{p})
$$

One sees that in contrast to the relative particle density fluctuations given by eq. (5), the energy density fluctuations (7) depend not only on the average energy density but on the on the energy second moment, i.e. $\left\langle e^{2}\right\rangle$, as well. 
As already mentioned, our approach is classical. There are two quantum effects: particle localization and bosonic or fermionic statistics which should be considered before the approach is applied to a quantum system. If the cell size $\Delta x$ is much larger than the average length of particle de Broglie wave $\lambda$, i.e.

$$
\Delta x \gg \lambda,
$$

the particles can be treated as well localized. One also finds analogous requirement for $\Delta t$, but for the ultrarelativistic system under consideration, the space and time scales, as the momentum and energy ones, are usually of the same order. Thus, we further discuss only the condition (9), which is checked below for the two cases: the equilibrium quark-gluon plasma and the parton system from the early stage of ultrarelativistic heavy-ion collisions.

One takes into account the effect of quantum statistics substituting the distribution function $f$ by $f(1 \pm f)$, where + is for bosons and - for fermions, in eq. (2)). For the equilibrium quark-plasma the numerical effect of the statistics is small because the quark (fermionic) and gluon (bosonic) corrections, which are of comparable value, act in the opposite directions. In the case of the parton system from the early stage of ultrarelativistic heavy-ion collisions, where gluons dominate, we also neglect the effect of bosonic statistics. The correction is expected to be small but significantly complicates the computation. The point is that the parton density is large at the early stage of the collision but the phase-space density, which matters for the quantum statistics effects, is reduced due to the large longitudinal momentum range of the partons.

The quarks and gluons are taken as noninteracting in our approach. More specifically, the partons are assumed to follow the straight line trajectories in eq. (2). Since the QCD color forces are of long range, the quarks and gluons always interact in a many-parton system. However, the effect of interaction is minor at the space scale which is much smaller than the inverse momentum transfer due to the interaction. Therefore, our formulas (5) and (7) are basically correct as long as

$$
\Delta x \ll \frac{1}{q},
$$

where $q$ is the characteristic momentum transfers discussed below.

To obtain our final formulas (5) and (7) the partons have been assumed to be massless. This assumption is correct for the case of equilibrium plasma at the sufficiently large temperature when the interaction can be treated as a small perturbation. The situation is less clear when the early stage of nucleus-nucleus collision is considered. Then, the partons are off mass-shell. Nevertheless we still treat them as massless i.e. we assume that the parton mass ('offeshellness') is smaller than $1 / \Delta x$. We adopt this assumption keeping in mind that the partons with the 'offshellness' $\mu$ decay after $\mu^{-1}$. Thus, they can be treated as noninteracting at a much smaller scale only.

After these comments let us discuss the equilibrium quark-gluon plasma. The distribution function then reads

$$
f^{\mathrm{eq}}(\mathbf{p})=\frac{g_{g}}{e^{\beta E_{p}}-1}+\frac{g_{q}}{e^{\beta E_{p}}+1},
$$


where $\beta^{-1}=T$ is the temperature and $g_{g}=16$ and $g_{q}=24$ are the numbers of the internal degrees of freedom of quarks (of two flavours) and gluons within the $\mathrm{SU}(3)$ gauge group. The plasma is assumed to be baryonless and the quarks, as gluons, are massless.

Substituting the function (11) to eqs. (11,6) and (8) we get, respectively,

$$
\begin{gathered}
\langle\rho\rangle=\frac{34 \zeta(3)}{\pi^{2}} T^{3} \cong 4.14 T^{3}, \\
\langle\varepsilon\rangle=\frac{37 \pi^{2}}{30} T^{4} \cong 12.2 T^{4}, \\
\left\langle e^{2}\right\rangle=\frac{462 \zeta(5)}{\pi^{2}} T^{5} \cong 48.5 T^{5},
\end{gathered}
$$

with $\zeta(z)$ being the zeta Riemann function; $\zeta(3) \cong 1.202$ and $\zeta(5) \cong 1.037$. The relative fluctuations of particle and energy densities (5) and (7) then are

$$
\begin{aligned}
& \sqrt{\frac{\left\langle\rho^{2}\right\rangle-\langle\rho\rangle^{2}}{\langle\rho\rangle^{2}}} \cong \frac{0.124}{\Delta x^{3 / 2} T^{3 / 2}}, \\
& \sqrt{\frac{\left\langle\varepsilon^{2}\right\rangle-\langle\varepsilon\rangle^{2}}{\langle\varepsilon\rangle^{2}}} \cong \frac{0.144}{\Delta x^{3 / 2} T^{3 / 2}},
\end{aligned}
$$

where we put for simplicity $\Delta t=0$.

We estimate the average length of particle de Broglie wave as the inverse average momentum, which in turn is defined as $\sqrt{\left\langle\left\langle\mathbf{p}^{2}\right\rangle\right\rangle}$, where $\langle\langle\ldots\rangle\rangle$ denotes averaging over particles. Therefore,



and the condition (9) gets the form $\Delta x T \gg 0.3$.

In the equilibrium quark-gluon plasma the characteristic momentum transfer can be identified with the Debye screening mass $m_{D}$, which for two flavours and three colors equals $g T$ with $g$ being the QCD coupling constant, see e.g. [四]. Then, the condition (10) is $\Delta x T \ll 1 / g$.

One sees that the two conditions, which allows us to treat partons as free and classical, can be fulfilled simultaneously if $g^{-1} \gg 1$ i.e. when the plasma is weakly interacting. Unfortunately, at the temperatures of order of a few hundreds $\mathrm{MeV} g$ is not much smaller than unity. Therefore, $\Delta x$ should be close to $T^{-1}$ that the two conditions are not badly violated.

The result (12) can be helpful in choosing the physically reasonable size of the elementary fluid cell in the numerical hydrodynamical calculations. On one hand, the cell should small enough to get details of the density profiles, but on the other hand, the cell size should be sufficiently large to reduce the fluctuations. One sees from eq. (12) that at $T=200 \mathrm{MeV}$ and $\Delta x=1 \mathrm{fm}$ the energy density deviates from the average value by about $14 \%$. The fluctuations increase to $41 \%$ for $\Delta x=0.5$ 
fm. However, such a small cell is at the border line of applicability of our classical approach.

The size of the space-time cell is sometimes dictated by the characteristic scale of the phenomenon under considerations. When the deconfinement phase transition is discussed, the cell size $\Delta x$ should be identified with the inverse confinement scale parameter $\Lambda_{Q C D} \cong 200 \mathrm{MeV}$. At the critical temperature $T_{c}=150 \mathrm{MeV}$ the energy density fluctuations are about $22 \%$. Such sizeable fluctuations can significantly speedup the process of hadronization. However, one should keep in mind that near the phase transition the quark-gluon plasma is no longer a system of weakly interacting particles and the formulas derived above can provide only a very rough estimate.

Let us now consider the nonequilibrium plasma from the early stage of ultrarelativistic heavy-ion collisions. It is commonly believed that the perturbative processes, which are under theoretical control, play an important, if not dominant, role in these collisions, see e.g. [6]. We adopt this conventional point of view and discuss density fluctuations of hard and semihard partons which are characterized by the transverse momentum being relatively large, at least $2 \mathrm{GeV}$, when compared to the QCD scale parameter $\Lambda_{Q C D}$.

We estimate the particle (mostly gluons) density taking the numbers from [5], where it has been found that about 570 perturbative gluons are generated at the early stage of the central $\mathrm{Au}-\mathrm{Au}$ collision at RHIC $(\sqrt{s}=200 \mathrm{GeV}$ per N-N collision) and 8100 at $\mathrm{LHC}(\sqrt{s}=6 \mathrm{TeV}$ per N-N collision). Assuming that all these gluons appear in the cylinder of the volume $\pi r_{0}^{2} A^{2 / 3} l$, where $r_{0}=1.1 \mathrm{fm}, A=197$ and $l=1 \mathrm{fm}$, we get the average densities

$$
\langle\rho\rangle \cong\left\{\begin{array}{lll}
4.4 \mathrm{fm}^{-3} & \text { for } & \text { RHIC }, \\
63 \mathrm{fm}^{-3} & \text { for } & \text { LHC },
\end{array}\right.
$$

which immediately provide (via eq. (5)) the density fluctuations.

To estimate the energy density fluctuations one needs the parton momentum distribution. We take it in the form which corresponds to the flat rapidity distribution in the interval $(-Y, Y)$ i.e.

$$
f(\mathbf{p})=\frac{1}{2 Y} \Theta(Y-y) \Theta(Y+y) h\left(p_{\perp}\right) \frac{1}{p_{\perp} \operatorname{ch} y},
$$

where $y$ and $p_{\perp}$ denote the parton rapidity and transverse momentum. We do not specify the transverse momentum distribution $h\left(p_{\perp}\right)$ because it is sufficient for our considerations to demand that the distribution (13) is strongly elongated along the beam axis i.e. $e^{Y} \gg 1$.

The QCD-based computations, see e.g. [6], show that the rapidity distribution of partons produced at the early stage of heavy-ion collisions is essentially gaussian with the width of about one to two units. When the distribution (13) is used to simulate the gaussian one, $Y$ does not measure the size of the 'plateau' but rather the range over which the partons are spread. If one takes the gaussian distribution of the variance $\sigma$ and the distribution (13) of the same variance, then $Y=\sqrt{3} \sigma$. 
One computes the average energy (6) and energy squared (8) densities with the distribution (13) as

$$
\begin{aligned}
\langle\varepsilon\rangle & =\frac{\operatorname{sh} Y}{Y}\left\langle p_{\perp}\right\rangle\langle\rho\rangle \cong \frac{e^{Y}}{2 Y}\left\langle p_{\perp}\right\rangle\langle\rho\rangle \\
\left\langle e^{2}\right\rangle & =\frac{\operatorname{sh} 2 Y+2 Y}{4 Y}\left\langle p_{\perp}^{2}\right\rangle\langle\rho\rangle \cong \frac{e^{2 Y}}{8 Y}\left\langle p_{\perp}\right\rangle^{2}\langle\rho\rangle
\end{aligned}
$$

and gets the energy density fluctuations

$$
\sqrt{\frac{\left\langle\varepsilon^{2}\right\rangle-\langle\varepsilon\rangle^{2}}{\langle\varepsilon\rangle^{2}}} \cong \frac{0.178}{\Delta x^{3 / 2}} \sqrt{\frac{Y}{\langle\rho\rangle}},
$$

where $\Delta t=0$.

Taking the values of $Y$ given in [5] i.e. $Y \cong 2.5$ at RHIC and $Y \cong 5.0$ at LHC and the gluon density, which has been estimated above, one gets the energy density fluctuations. Eq. (14) tells us that the fluctuations at RHIC are $13 \%$ for $\Delta x=1$ fm and increase to $38 \%$ for $\Delta x=0.5 \mathrm{fm}$. At LHC the fluctuations are smaller by factor 2.7. Since the average transverse momentum of perturbative partons is of $\mathrm{GeV}$ order and the longitudinal momentum is even larger, their wavelength is a small fraction of $1 \mathrm{fm}$. The condition (9) is then easily satisfied for the cell size of interest. Neglecting of the interaction seems to be reasonable as well, when the partons with small 'offshellness' are taken into account.

Let us summarize our considerations. We have derived the formulas which describe how the particle and energy densities fluctuate in a space-time cell of the volume $\Delta x^{3} \Delta t$. These formulas, which get a very simple form for massless particles, have been applied to estimate the density fluctuations in the quark-gluon plasma. We have considered the equilibrium plasma and the anisotropic parton system from the early stage of ultrarelativistic heavy-ion collisions at RHIC or LHC. In both cases the fluctuations can be large within the reasonable values of the parameters of interest. Sizeable density fluctuations are of physical interest when the temporal evolution of the plasma system is studied. As shown in [2], the hydrodynamics is then noticeably modified. The density fluctuations seem to be even more important when the hadronization is analysed or one considers the processes, such as the $J / \psi$ dissociation in the plasma [7], which are strongly density dependent.

I am very grateful to Mark. I. Gorenstein for critical reading of the manuscript. 


\section{References}

[1] St. Mrówczyński, Phys. Lett. B393 (1997) 26; ibid B314, 118 (1993); Phys. Rev. C49, 2191 (1994).

[2] M. Gyulassy, D. Rischke, and B. Zhang, Nucl. Phys. A613, 397 (1997).

[3] A.I. Akhiezer, I.A. Akhiezer, R.V. Polovin, A.G. Sitenko, and K.N. Stepanov, Plasma Electrodynamics (Pergamon, New York, 1975).

[4] St. Mrówczyński, in Quark-Gluon Plasma, ed. R. Hwa (World Scientific, Singapore, 1990).

[5] T.S. Biró, B. Müller, and X.-N. Wang, Phys. Lett. B283, 171 (1992).

[6] K. Geiger, Phys. Rep. 258, 237 (1995); X.-N. Wang, Phys. Rep. 280, 287 (1997).

[7] D. Kharzeev, Nucl. Phys. A610, 418c (1996). 